\title{
Optimization of Guaranteed Results under Functional Restrictions on the Dynamic Disturbance
}

\author{
D. A. Serkov ${ }^{a, b}$ \\ Presented by Academician A. V. Kryazhimskii October 22, 2012
}

Received December 12, 2012

DOI: $10.1134 / \mathrm{S} 1064562413030113$

1. This work is related to the theory of guaranteeing position control (see [1, 2]) and relies on the results of [3-5]. We examine the properties of an optimal guaranteed result and describe methods for constructing an optimal control strategy in the case when an unobservable dynamic disturbance acting on the control system is subject to a priori functional restrictions.

Control problems with functional restrictions imposed on the input dynamic disturbance have numerous interpretations and have been studied in various formalizations. In $[6,7]$ the properties of linear control systems were compared in the case of disturbances specified by previously unknown but fixed functions of time (programmed disturbances), disturbances generated by continuous feedback, and disturbances formed by upper semicontinuous set-valued position strategies. In [5], assuming that the disturbances with a priori restrictions imposed on their values are contained in a previously unknown $L_{2}$-compact set, it was shown that the optimal guaranteed result achieved in the class of position strategies with full memory is equal to that achieved in the class of quasi-strategies, i.e., nonanticipating program responses to disturbance realizations [2].

In this work, the problem is studied in the formulation proposed in [5]. The result obtained is stronger than that of [5] concerning the unimprovability of position strategies with full memory in the case of a continuous cost functional.

2. Consider a control system governed by the differential equation

$$
\dot{x}(t)=f(t, x(t), u(t), v(t))
$$

\footnotetext{
${ }^{a}$ Krasovskii Institute of Mathematics and Mechanics, Ural Branch, Russian Academy of Sciences, ul. S. Kovalevskoi 16, Yekaterinburg, 620990 Russia

${ }^{b}$ Ural Federal University, ul. Lenina 51, Yekaterinburg, 620083 Russia e-mail:serkov@imm.uran.ru,d.a.serkov@gmail.com
}

in $\mathbb{R}^{n}$ on a time interval $T \triangleq\left[t_{0}, \vartheta\right]\left(t_{0}<\vartheta\right)$ with the initial condition $x\left(t_{0}\right)=x_{0}$. Here, $\triangleq$ denotes "being equal by definition"; $x_{0} \in \mathbb{R}^{n} ; u(\cdot)$ and $v(\cdot)$ are an admissible control and an admissible disturbance defined as arbitrary Borel measurable functions from $T$ to given compact sets $\mathscr{P} \subset \mathbb{R}^{p}$ and $\mathscr{Q} \subset \mathbb{R}^{q}$, respectively; and $f(\cdot)$ : $T \times \mathbb{R}^{n} \times \mathscr{P} \times \mathscr{2} \mapsto \mathbb{R}^{n}$ is a continuous function satisfying (with respect to the second variable) the sublinear growth condition and a local Lipschitz condition with growth and Lipschitz constants that are uniform with respect to the other variables (see [8, Section II.4]). The sets of all admissible controls and all admissible disturbances are denoted by $\mathcal{U}$ and $\mathscr{V}$, respectively.

Let $x\left(\cdot, x_{0}, u(\cdot), v(\cdot)\right)$ denote the motion of the system from the state $x_{0} \in G_{0}$ under the action of an admissible control $u(\cdot)$ and an admissible disturbance $v(\cdot)$, i.e., the solution in the sense of Carathéodory on $T$ of Eq. (1) with the initial condition $x\left(t_{0}\right)=x_{0}$ (the above assumptions ensure the existence and uniqueness of this motion).

As is customary (see [1]), we assume that the controller influences the motion from the initial position $x_{0}$ by generating admissible control values. Let $\gamma(\cdot)$ be a continuous functional on $C\left(T, \mathbb{R}^{n}\right)$ (cost functional). The goal of the controller is to minimize the largest (worst) of the cost functional values occurring when the system is driven by the generated admissible control and any admissible disturbance.

Current control values are generated discretely as based on the current history of the motion and the control. As usual (see [1]), we assume that the current disturbance values cannot be observed in the control process.

The above methods for generating current admissible control values are formalized as position strategies with full memory. Let us give the rigorous definition (see [5]). The partition (of the time interval $T$ ) is any finite set $\Delta \triangleq\left(\tau_{i}\right)_{i=0}^{m}$ from $T$ such that $t_{0}=\tau_{0}<\tau_{1}<\ldots<$ $\tau_{m}=\vartheta$. Let $\mathbf{d}(\Delta) \triangleq \max _{i \in 1, \ldots, m}\left(\tau_{i}-\tau_{i-1}\right)$ (here and below, 
$i, \ldots, j$ denotes the following segment of the set of nonnegative integers: $\{i, i+1, \ldots, j\}, i, j \in \mathbb{N} \cup\{0\})$. The set of all partitions is denoted by $D$. The feedback with full memory on a partition $\Delta=\left(\tau_{i}\right)_{i=0}^{m}$ is any family $\mathbf{U} \triangleq$ $\left(\mathbf{U}_{i}(\cdot)\right)_{i=0}^{m-1}$, where $\mathbf{U}_{i}(\cdot): C\left(\left[t_{0}, \tau_{i}\right], \mathbb{R}^{n}\right) \mapsto \mathscr{P}(i \in 0, \ldots$, $(m-1))$. The position strategy with full memory is any family $\mathbf{U}=\left(\mathbf{U}^{\Delta}\right)_{\Delta \in D}$, where, for each $\Delta \in D, \mathbf{U}^{\Delta}$ is feedback with full memory on the partition $\Delta$.

The motion from the state $x_{0} \in \mathbb{R}^{n}$ generated by the full-memory feedback $\mathbf{U}=\left(\mathbf{U}_{i}(\cdot)\right)_{i=0}^{m-1}$ on $\Delta$ and by an admissible disturbance $v(\cdot)$ is a function $x(\cdot)=x\left(\cdot, x_{0}\right.$, $\Delta, \mathbf{U}, v(\cdot))$ defined by the condition $x(\cdot)=x\left(\cdot, x_{0}, u(\cdot)\right.$, $\mathrm{v}(\cdot)), u(t)=\mathbf{U}_{i}\left(x(\cdot)_{\tau_{i}}\right)$ for all $t \in\left(\tau_{i}, \tau_{i+1}, i \in 0, \ldots .\right.$, $(m-1)$. Here and below, $z(\cdot)_{\tau}$ is the restriction to $\left[t_{0}, \tau\right]$ $(\tau \in T)$ of a function $z(\cdot)$ defined on an interval that belongs to $T$ and contains $\left[t_{0}, \tau\right]$.

For each nonempty $V \subset \mathscr{V}$, each $x_{0} \in \mathbb{R}^{n}$, and each full-memory position strategy $\mathbf{U}=\left(\mathbf{U}^{\Delta}\right)_{\Delta \in D}$, let $X\left(x_{0}\right.$, $\mathbf{U}, V)$ denote the set of all functions $x(\cdot) \in C\left(T, \mathbb{R}^{n}\right)$, each being the limit in $C\left(T, \mathbb{R}^{n}\right)$ of the sequence $\left(x\left(\cdot, x_{0 k}, \Delta_{k}, \mathbf{U}^{\Delta_{k}}, v_{k}(\cdot)\right)_{k=1}^{\infty}\right.$ for some $x_{0 k} \in \mathbb{R}^{n}, \Delta_{k} \in D$, $v_{k}(\cdot) \in V(k \in \mathbb{N})$ such that $x_{0 k} \rightarrow x_{0}$ and $\mathbf{d}\left(\Delta_{k}\right) \rightarrow 0$.

In what follows, $\operatorname{comp}(\mathscr{V})$ denotes the set of all subsets of $\mathscr{V}$ that are compact in $L_{2}\left(T, R^{q}\right)$. For each $x_{0} \in$ $\mathbb{R}^{n}$ and each full-memory position strategy $\mathbf{U}$, we define

$$
\begin{gathered}
\mathscr{X}\left(x_{0}, \mathbf{U}\right) \stackrel{\Delta}{=} X\left(x_{0}, \mathbf{U}, \mathscr{V}\right), \\
\mathscr{X}_{c}\left(x_{0}, \mathbf{U}\right) \triangleq \\
\bigcup_{V \in \operatorname{comp}(\mathscr{V})} X\left(x_{0}, \mathbf{U}, \mathbf{V}\right) .
\end{gathered}
$$

Let $\mathscr{Y}$ be the set of all full-memory position strategies. Given $x_{0} \in \mathbb{R}^{n}$, the quantities

$$
\begin{aligned}
& \Gamma\left(x_{0}\right) \triangleq \inf _{\mathbf{U} \in \mathscr{S}} \sup _{x(\cdot) \in \mathscr{X}\left(x_{0}, \mathbf{U}\right)} \gamma(x(\cdot)), \\
& \Gamma_{c}\left(x_{0}\right) \triangleq \inf _{\mathbf{U} \in \mathscr{S}} \sup _{x(\cdot) \in \mathscr{x}_{c}\left(x_{0}, \mathbf{U}\right)} \gamma(x(\cdot))
\end{aligned}
$$

are called the optimal guaranteed results in the class of full-memory position strategies in the initial state $x_{0}$ with no functional restrictions on the disturbances and with $L_{2}$-compact restrictions on the disturbances, respectively.

A strategy $\mathbf{U}_{*} \in \mathscr{Y}$ is said to be optimal in the initial state $x_{0} \in \mathbb{R}^{n}$ with no functional restrictions on the disturbances and with $L_{2}$-compact restrictions on the disturbances if

$$
\begin{aligned}
\sup _{x(\cdot) \in \mathscr{X}\left(x_{0}, \mathbf{U}_{*}\right)} \gamma(x(\cdot)) & =\Gamma\left(x_{0}\right), \\
\sup _{x(\cdot) \in \mathscr{X}_{c}\left(x_{0}, \mathbf{U}_{*}\right)} \gamma(x(\cdot)) & =\Gamma_{c}\left(x_{0}\right),
\end{aligned}
$$

respectively.
Following [2, p. 24], the quasi-strategy is any mapping $\alpha(\cdot): \mathscr{V} \mapsto \mathcal{Q}$ such that $\alpha(v(\cdot))_{\tau}=\alpha\left(v^{\prime}(\cdot)\right)_{\tau}$ for all $\tau \in T$ and $v(\cdot), v^{\prime}(\cdot) \in \mathscr{V}$ satisfying $v(\cdot)_{\tau}=v^{\prime}(\cdot)_{\tau}$. For any $x_{0} \in \mathbb{R}^{n}$ and any quasi-strategy $\alpha(\cdot)$, the elements of the set

$$
\mathscr{L}\left(x_{0}, \alpha(\cdot)\right) \triangleq\left\{x\left(\cdot, x_{0}, \alpha(v(\cdot)), v(\cdot)\right) \mid v(\cdot) \in \mathscr{V}\right\}
$$

are the motions from $x_{0}$ generated by $\alpha(\cdot)$. Let $\mathbf{Q}$ denote the set of all quasi-strategies. The optimal guaranteed result in the initial state $x_{0} \in \mathbb{R}^{n}$ in the class of quasi-strategies (with no functional restrictions on disturbances) is defined as

$$
\Gamma_{q}\left(x_{0}\right) \triangleq \inf _{\alpha(\cdot) \in \mathbf{Q}} \sup _{x(\cdot) \in \mathscr{L}\left(x_{0}, \alpha(\cdot)\right)} \gamma(x(\cdot)) .
$$

In a similar manner, we can define the optimal guaranteed result in the class of quasi-strategies with $L_{2}$-compact restrictions on disturbances. However, these definitions imply that the defined quantities are equal to each other: from the point of view of the optimal guaranteed result, quasi-strategies are insensitive to functional restrictions on disturbances.

Theorem 1. For every $x_{0} \in \mathbb{R}^{n}$,

$$
\Gamma_{q}\left(x_{0}\right) \leq \Gamma_{c}\left(x_{0}\right) \leq \Gamma\left(x_{0}\right) .
$$

It follows from the results of [2] that both inequalities in (5) become equalities for every $x_{0} \in \mathbb{R}^{n}$ if the saddle point condition holds, namely, if

$$
\begin{aligned}
& \min _{u \in \mathscr{P}} \max \in \mathcal{L} \\
= & \max _{v \in \mathcal{2}} \min _{u \in \mathscr{P}}\langle l, f(t, x, u, v)\rangle \\
& \langle t, x, u, v)\rangle
\end{aligned}
$$

for all $t \in T$ and $l, x \in \mathbb{R}^{n}$. Here and below, $\langle\cdot, \cdot\rangle$ denotes the scalar product in $\mathbb{R}^{n}$. Generally speaking, the last condition is not assumed to hold. In this situation, some of the inequalities in (5) can be strict. Examples of situations in which the first and last elements in (5) differ are well known in the theory of guaranteeing control (see [2, Chapter VI, Section 1]).

For a cost functional $\gamma$ that is uniformly $\left(L^{1}, \delta\right)$ continuous on the set of all motions of system (1), an example with the second inequality in (5) being strict was given in [5]. For a continuous functional, an example of a similar inequality is presented at the end of this paper.

3 . Since the least of the optimal guaranteed results written in (5) is the optimal guaranteed result in the class of quasi-strategies, of special interest are those functional restrictions on disturbances $\left(L_{2}\right.$-compact restrictions in this work) and those conditions under which the corresponding optimal guaranteed result in the class of full-memory position strategies coincides with that in the class of quasi-strategies. In this case, the class of full-memory position strategies is unimprovable (in the given initial state) in the sense that the use of any past and current values of admissible disturbances in the generation of an admissible control does not improve the guaranteed result. 
According to [5], a sufficient condition for the unimprovability of the class of position strategies with memory under $L_{2}$-compact restrictions on disturbances is that the mapping $V \mapsto f(t, x, u, v)$ is one-toone for all $(t, x, u) \in T \times \mathbb{R}^{n} \times \mathscr{P}$. The goal of this paper is to establish a more general sufficient condition and present the corresponding strategy from $\mathscr{Y}$.

We begin with defining a special strategy $\mathbf{U}_{*} \in \mathscr{Y}$. Its construction relies on sets of motions generated by "almost optimal" quasi-strategies. Specifically, for every $x_{0} \in \mathbb{R}^{n}$ and every $\varepsilon>0$, let

$$
\begin{gathered}
\mathbf{Q}_{\varepsilon}\left(x_{0}\right) \triangleq\left\{\alpha(\cdot) \in \mathbf{Q} \sup _{x(\cdot) \in X\left(x_{0}, \alpha(\cdot)\right)} \gamma(x(\cdot)) \leq \Gamma_{q}\left(x_{0}\right)+\varepsilon\right\}, \\
\mathscr{W}\left(x_{0}\right) \triangleq \bigcap_{\varepsilon>0} \mathrm{cl} \bigcup_{\alpha(\cdot) \in \mathbf{Q}_{\varepsilon}\left(x_{0}\right)} \mathscr{X}\left(x_{0}, \alpha(\cdot)\right),
\end{gathered}
$$

where cl denotes the closure of a set in $C\left(T, \mathbb{R}^{n}\right)$. For an arbitrary $\tau \in T$, we define $\mathcal{W}\left(x_{0}\right)_{\tau} \triangleq\left\{x(\cdot)_{\tau} \mid x(\cdot) \in\right.$ $\left.\mathcal{W}\left(x_{0}\right)\right\}$, and, for every $y(\cdot) \in C\left(\left[t_{0}, \tau\right], \mathbb{R}^{n}\right)$, we fix the element $w(\cdot \mid \tau, y(\cdot)) \in \mathcal{W}\left(y\left(t_{0}\right)\right)_{\tau}$ nearest to $y(\cdot)$ in $C\left(\left[t_{0}, \tau\right], \mathbb{R}^{n}\right)$.

Now let us define the position strategy $\mathbf{U}_{*}$ of interest. The feedback $\mathbf{U}_{*}^{\Delta}=\left(\mathbf{U}_{* i}^{\Delta}(\cdot)\right)_{i=0}^{m-1}$ is defined inductively on the partition $\Delta=\left(\tau_{i}\right)_{i=0}^{m}$ with a constant step.

Given some $\bar{u}_{0} \in \mathscr{P}$, for every $x_{0}(\cdot) \in C\left(\left[t_{0}, \tau_{0}\right], \mathbb{R}^{n}\right)$ (recall that $\tau_{0}=t_{0}$ ), we set $\mathbf{U}_{*_{0}}^{\Delta}\left(x_{0}(\cdot)\right)=\bar{u}_{0}$. Additionally, we introduce a constant admissible control $\bar{u}_{0}(\cdot)$ with the value equal to $\bar{u}_{0}$.

For any $x_{1}(\cdot) \in C\left(\left[t_{0}, \tau_{1}\right], \mathbb{R}^{n}\right)$, before specifying $\mathbf{U}_{* 1}^{\Delta}\left(x_{1}(\cdot)\right) \in \mathscr{P}$, we define the auxiliary vector $\bar{V}_{0}^{\Delta}=$ $\bar{v}_{0}^{\Delta}\left(x_{1}(\cdot), u_{0}\right) \in 2$ on the time interval $\left[t_{0}, \tau_{1}\right]$. This vector approximates the values of the unobservable disturbance occurring in the formation of the motion $x_{1}(\cdot)$ of the system on the interval $\left[\tau_{0}, \tau_{1}\right]$ and is found by solving a suitable inverse dynamics problem (see [3, 4]). Additionally, we specify a vector $y_{0}\left(\tau_{0}\right) \in \mathbb{R}^{n}$ used as an initial state of the auxiliary motion $y_{0}(\cdot)$ modeling the optimal behavior of system (1) under the auxiliary disturbance $\bar{V}_{0}^{\Delta}$ on interval $\left[\tau_{0}, \tau_{1}\right]$. The value $\mathbf{U}_{* 1}^{\Delta}\left(x_{1}(\cdot)\right)$ plays the role of an admissible control on $\left[\tau_{1}, \tau_{2}\right]$ formed as based on the memory $x_{1}(\cdot)$ and is chosen to be the optimal response (in the formalism of counterstrategies [1]) to the disturbance value $\bar{v}_{0}^{\Delta}$ in the auxiliary motion $y_{0}(\cdot)$. Specifically, we set

$$
\begin{gathered}
\bar{V}_{0}^{\Delta} \in \underset{v \in 2}{\operatorname{argmin}}\left|f\left(\tau_{0}, x_{1}\left(\tau_{0}\right), \bar{u}_{0}, v\right)-\frac{x_{1}\left(\tau_{1}\right)-x_{1}\left(\tau_{0}\right)}{\tau_{1}-\tau_{0}}\right|, \\
y_{0}\left(\tau_{0}\right) \triangleq x_{1}\left(t_{0}\right),
\end{gathered}
$$

$$
\mathbf{U}_{* 1}^{\Delta}\left(x_{1}(\cdot)\right)
$$

$\in \underset{u \in \mathscr{P}}{\arg \min }\left\langle y_{0}\left(\tau_{0}\right)-w\left(\tau_{0} \mid \tau_{0}, y_{0}\left(\tau_{0}\right)\right), f\left(\tau_{0}, y_{0}\left(\tau_{0}\right), u, \bar{v}_{0}\right)\right\rangle$.

Here and below, $|\cdot|$ denotes the Euclidean norm. Assume that, for a certain $i \in 1, \ldots,(m-1)$, the values $\mathbf{U}_{* k}^{\Delta}\left(x_{k}(\cdot)\right) \in \mathscr{P}$ are determined for all $k \in 0, \ldots, i$ and $x_{k}(\cdot) \in C\left(\left[t_{0}, \tau_{k}\right], \mathbb{R}^{n}\right)$. For any $x_{i+1}(\cdot) \in C\left(\left[t_{0}, \tau_{i}\right], \mathbb{R}^{n}\right)$, before specifying $\mathbf{U}_{*_{i+1}}^{\Delta}\left(x_{i+1}(\cdot)\right) \in \mathbb{R}^{n}$, we specify the vectors $\bar{V}_{i}^{\Delta} \triangleq \bar{V}_{i}^{\Delta}\left(x_{i+1}(\cdot), u_{i}(\cdot)\right) \in 2$ and $y_{i}\left(\tau_{i}\right) \in \mathbb{R}^{n}$ (which have the same meaning as their counterparts defined above in the case $i=1$ ). Let

$$
\begin{gathered}
\bar{V}_{i}^{\Delta} \in \underset{v \in 2}{\operatorname{argmin}} \mid f\left(\tau_{i}, x_{i+1}\left(\tau_{i}\right), \mathbf{U}_{* i}^{\Delta}\left(x_{i+1}(\cdot)_{\tau_{i}}\right), v\right) \\
-\frac{x_{i+1}\left(\tau_{i+1}\right)-x_{i+1}\left(\tau_{i}\right)}{\tau_{i+1}-\tau_{i}} \mid, \\
y_{i}\left(\tau_{i}\right)=y_{i-1}\left(\tau_{i-1}\right)+\int_{\tau_{i-1}}^{\tau_{i}} f\left(t, y_{i}(t), \mathbf{U}_{* i}^{\Delta}\left(x_{i+1}(\cdot)_{\tau_{i}}\right), \bar{V}_{i}^{\Delta}\right) d t, \\
\mathbf{U}_{* i+1}^{\Delta}\left(x_{i+1}(\cdot)\right) \\
\in \underset{u \in \mathscr{P}}{\operatorname{argmin}}\left\langle y_{i}\left(\tau_{i}\right)-w\left(\tau_{i} \mid \tau_{i}, y_{i}(\cdot)\right), f\left(\tau_{i}, y_{i}\left(\tau_{i}\right), u, \bar{v}_{i}^{\Delta}\right)\right\rangle .
\end{gathered}
$$

The feedback $\mathbf{U}_{*}^{\Delta}=\left(\mathbf{U}_{*_{i}}^{\Delta}(\cdot)\right)_{i=0}^{m-1}$ on a uniform partition $\Delta$ has been defined. The definition of the feedback $\mathbf{U}_{*}^{\Delta}$ on an arbitrary partition $\Delta$ differs by insignificant technical details. The full-memory position strategy of interest is $\mathbf{U}_{*} \triangleq\left(\mathbf{U}_{*}^{\Delta}\right)_{\Delta \in D}$.

For $(t, x) \in T \times \mathbb{R}^{n}$ and $u \in \mathscr{P}$, we introduce the quotient set $2 /{ }_{(t, \tilde{x}, u)}$ of a set 2 generated by the equivalence relation ${ }_{(t, \tilde{x}, u)}$ :

$$
\begin{aligned}
& \left(v_{1_{(t, \tilde{x}, u)}} V_{2}\right) \Leftrightarrow\left(f\left(t, x, u, v_{1}\right)=f\left(t, x, u, v_{2}\right)\right), \\
& v_{1}, v_{2} \in 2 \text {. }
\end{aligned}
$$

Theorem 2. Suppose that, for system (1), the quotient sets $\mathscr{2} /(t, \tilde{x}, u)$ are independent of $u \in \mathscr{P}$ :

$$
\begin{gathered}
\underset{(t, x, u)}{2 / \sim} \underset{\left(t, x, u^{\prime}\right)}{2 / \sim} \text { for all } u, u^{\prime} \in \mathscr{P}, \\
(t, x) \in T \times \mathbb{R}^{n} .
\end{gathered}
$$

Then, for any $x_{0} \in \mathbb{R}^{n}$, we have $\Gamma_{q}\left(x_{0}\right)=\Gamma_{c}\left(x_{0}\right)$ and the strategy $\mathbf{U}_{*}$ is optimal in the initial state $x_{0}$ under $L_{2}$-compact restrictions on disturbances.

An example of control system (1) satisfying condition (9) is the system

$$
\begin{gathered}
\dot{x}(t)=f(t, x(t), u(t)) \\
+g(t, x(t), u(t)) h(t, x(t), v(t)),
\end{gathered}
$$

where $f(\cdot): T \times \mathbb{R}^{n} \times \mathscr{P} \mapsto \mathbb{R}^{n}, h(\cdot): T \times \mathbb{R}^{n} \times \mathscr{Q} \mapsto \mathbb{R}^{m}$, $m \in \mathbb{N}, g(\cdot)$ maps $T \times \mathbb{R}^{n} \times \mathscr{P}$ to a normed space of 
matrices, and the kernel of the linear operator $g(t, x, u)$ : $\mathbb{R}^{m} \mapsto \mathbb{R}^{n}$ is independent of $u \in \mathscr{P}$ for all $(t, x) \in T \times \mathbb{R}^{n}$.

4. In some problems, the above construction of a strategy with full memory leads to efficient computational procedures. When system (8) is given by the equation

$$
\dot{x}(t)=A(t) x(t)+g(t, u(t), v(t)),
$$

and the performance index has the form $\gamma(x(\cdot)) \triangleq$ $\sigma(x(\vartheta))$, where $\sigma(\cdot): \mathbb{R}^{n} \mapsto \mathbb{R}$ is a convex and locally Lipschitz function, the form of the strategy $\mathbf{U}_{*}$ can be simplified if the regularity condition holds (see [1, Section 72; 2, Chapter III]). Specifically, let $\boldsymbol{\Phi}(\vartheta, s)$ be the fundamental matrix of system $(11), \sigma^{*}(\cdot): \mathbb{R}^{n} \mapsto \mathbb{R}$ be the adjoint of the function $\sigma(\cdot)$, and $\operatorname{dom}\left(\sigma^{*}(\cdot)\right) \subset \mathbb{R}^{n}$ be the effective set of $\sigma^{*}(\cdot)$. For arbitrary $(\tau, l) \in T \times \mathbb{R}^{n}$, we introduce

$$
\rho(\tau, l) \stackrel{\Delta}{=} \int_{\tau}^{\vartheta} \max \min \langle l, \boldsymbol{\Phi}(\vartheta, s) g(s, u, v)\rangle d s-\sigma^{*}(l)
$$

and define a counterstrategy [1, Section 86$] u_{0}(\cdot): T \times$ $\mathbb{R}^{n} \times \mathscr{Q} \mapsto \mathscr{P}$ by the conditions

$$
\begin{gathered}
u_{0}(\tau, x, v) \\
\in \underset{u \in \mathscr{P}}{\operatorname{argmin}}\left\langle l_{0}(\tau, x), \boldsymbol{\Phi}(\vartheta, \tau) g(\tau, u, v)\right\rangle, \\
l_{0}(\tau, x) \in \underset{l \in \operatorname{dom} \sigma^{*}(\cdot)}{\arg \max }[\langle l, \boldsymbol{\Phi}(\vartheta, \tau) x\rangle+\rho(\tau, l)], \\
(\tau, x, v) \in T \times \mathbb{R}^{n} \times 2 .
\end{gathered}
$$

Theorem 3. If condition (9) holds for system (11) and the function $\rho(\tau, \cdot)$ : $\operatorname{dom}\left(\sigma^{*}(\cdot)\right) \mapsto \mathbb{R}$ is concave for all $\tau \in T$, then $\Gamma_{q}\left(x_{0}\right)=\Gamma_{c}\left(x_{0}\right)$ for any $x_{0} \in \mathbb{R}^{n}$ and the strategy $\mathbf{U}_{* *}$ given by relations $(7),(8)$, and $\mathbf{U}_{* i+1}^{\Delta}\left(x_{i+1}(\cdot)\right) \triangleq$ $u_{0}\left(\tau_{i}, y_{i}\left(\tau_{i}\right), \bar{v}_{i}^{\Delta}\right)\left(\Delta=\left(\tau_{i}\right)_{i=0}^{m}, i \in 1, \ldots,(m-1)\right)$ is optimal in the initial state $x_{0}$ under $L_{2}$-compact restrictions on the disturbances.

Note that the strategies $\mathbf{U}_{*}$ and $\mathbf{U}_{* *}$ are universal; i.e., they are independent of the initial state $x_{0}$.

5. As an example, we consider the following control system of form (10):

$$
\begin{gathered}
\dot{x}_{1}(\tau)=u_{1}(\tau) \cdot v_{1}(\tau), \quad \tau \in T \triangleq[0,1], \\
\mathscr{P}=2 \triangleq\{-1,1\}, \\
\dot{x}_{2}(\tau)=g\left(x_{1}(\tau)\right) \cdot u_{2}(\tau) \cdot v_{2}(\tau), \\
g(x) \triangleq \max \{0, x\}, \quad x \in \mathbb{R},
\end{gathered}
$$

$$
\begin{gathered}
\left(x_{1}(0), x_{2}(0)\right)=(0,0), \quad u_{1}(\tau), u_{2}(\tau) \in \mathscr{P}, \\
V_{1}(\tau), v_{2}(\tau) \in \mathscr{2} .
\end{gathered}
$$

Let $\gamma(x(\cdot)) \triangleq x_{2}(1)\left(x(\cdot)=\left(x_{1}(\cdot), x_{2}(\cdot)\right) \in C\left(T, \mathbb{R}^{2}\right)\right)$. It can be verified that $\Gamma((0,0))=0.5$ and the conditions of Theorem 2 hold. At the same time, the right-hand side of the system is not injective with respect to $v_{2}$, i.e., does not satisfy the conditions of Theorem 9.1 in [5]. By applying Theorem 2, the optimal guaranteed result under $L_{2}$-compact restrictions on the disturbance can be easily obtained to be $\Gamma_{c}((0,0))=$ $\Gamma_{q}((0,0))=-0.5$. This example shows that the last inequality in (5) can also be strict in the case when the functional $\gamma$ is continuous in $C\left(T, \mathbb{R}^{n}\right)$.

\section{ACKNOWLEDGMENTS}

This work was supported by the Presidium of the Russian Academy of Sciences under the program "Dynamical Systems and Control Theory," by the Ural Branch of the Russian Academy of Sciences (project no. 12-P-1-1002), and by the Russian Foundation for Basic Research (project no. 12-01-00290).

\section{REFERENCES}

1. N. N. Krasovskii and A. I. Subbotin, Positional Differential Games (Nauka, Moscow, 1974) [in Russian].

2. A. I. Subbotin and A. G. Chentsov, Guaranty Optimization in Control Problems (Nauka, Moscow, 1981) [in Russian].

3. Yu. S. Osipov and A. V. Kryazhimskii, Inverse Problems for Ordinary Differential Equations: Dynamical Solutions (Gordon and Breach, London, 1995).

4. A. V. Kryazhimskii and Yu. S. Osipov, Izv. Akad. Nauk SSSR, Tekh. Kibern., No. 2, 51-60 (1983).

5. A. V. Kryazhimskii, "The Problem of Optimization of the Ensured Result: Unimprovability of Full-Memory Strategies," Constantin Carathéodory: An International Tribute (World Scientific, Teaneck, NJ, 1991), pp. 636675.

6. N. N. Barabanova and A. I. Subbotin, Prikl. Math. Mekh. 34, 796-803 (1970).

7. N. N. Barabanova and A. I. Subbotin, Prikl. Math. Mekh. 35, 385-392 (1971).

8. J. Warga, Optimal Control of Differential and Functional Equations (Academic, New York, 1972; Nauka, Moscow, 1982).

Translated by I. Ruzanova 